\title{
IGNITION OF A RECTANGULAR SOLID BY AN EXTERNAL HEAT FLUX*
}

\author{
CARLOS VÁZQUEZ-ESPÍ ${ }^{\dagger}$ AND AMABLE LIÑÁN ${ }^{\dagger}$
}

\begin{abstract}
The influence of corners on the ignition of a solid exposed to a surface energy flux is analyzed with large activation energy asymptotics. We begin with the analysis of the ignition of semi-infinite wedges by a constant heat flux. Two stages and two spatial zones, reactive and inert, are found. The ignition stage can be described by a slowly convergent asymptotic expansion for the increment in temperature due to the chemical reaction or, more accurately, by a simplified nonlinear parabolic equation to be solved numerically. This analysis applies to the ignition of two-dimensional finite bodies with corners if the external heat flux is large enough for the size of the reaction zone to be much smaller than that of the solid. The ignition of bodies with rectangular shape for small heat fluxes, when the reaction zone extends to the whole solid, is also analyzed.
\end{abstract}

Key words. combustion, ignition, thermal runaway, hot spot, activation energy asymptotics, Arrhenius kinetics

AMS subject classifications. 35C20, 35K57, 80A25

1. Introduction. Due to the strong sensitivity of the chemical reactions with temperature, a body of finite size can remain in a nonreactive or nearly nonreactive state if its temperature is low enough, so that the characteristic time associated with the chemical reaction is much larger than the characteristic time of the heat conduction process that removes heat off the system. If we subject the body to an external heat flux its temperature may rise to values such that the chemical time becomes shorter or, at least, comparable to the cooling time. Then one can expect to find ignition events, characterized by a sudden rise in temperature or thermal runaway, taking place at a hot spot at a well-defined time that is called ignition time.

In most studies on ignition of solids [1]-[7] the surface of the solid has been considered smooth. If the external flux is high enough the heating of the solid, when ignition occurs, will be confined to a thin surface layer, and the ignition delay will be independent of the body size and shape. However, it is reasonable to expect that the local heating of the solid will be accelerated, and the ignition time reduced, when the solid surface is rough, so that the heat from the external stimulus is concentrated in certain regions. A first study concerned with the influence of square corners on the ignition of solids, under an external heat flux, was given in [8], and extended to wedges in [9]; there, the ignition problem for a semi-infinite solid is treated from a numerical point of view. A formula correlating the ignition delay with the activation energy and the pre-exponential factor was obtained, as in [1], for a few values of the angle of the wedge. The ignition of wedges and cones was also analyzed in [10], highlighting the role of the inert distribution in determining the ignition time. An empirical ignition temperature was used and the ignition time, as a function of the angle of the wedge, was obtained by interpolation from the value corresponding to the half-space and from the numerical results of an approximate model for small values of the angle. These studies show a reduction of the ignition time due to the more rapid rise of the

* Received by the editors June 23, 1993; accepted for publication December 9, 1993. This research was supported by Comisión Interministerial de Ciencia y Tecnología grant ESP-90-0271.

† Escuela Técnica Superior de Ingenieros Aeronáuticos, Universidad Politécnica de Madrid, Ciudad Universitaria, 28040 Madrid, Spain. 
temperature at the edge of the wedge as compared with the rise taking place at the surface of the solid far from the edge.

The technique of large activation energy asymptotics has been widely used to describe the ignition process of one-dimensional solids [2]-[7] when the chemical reaction is modeled by an overall reaction of Arrhenius type, whose reaction rate is given by $\tilde{B} \exp (-E / R T)$. Here $\tilde{B}$ is the pre-exponential factor and $E$ is the activation energy, much larger than the thermal energy $R T$. We have recently used [11] these techniques to analyze the ignition of rectangular bodies under a step rise in surface temperature. We will present in this paper a large activation energy analysis of the ignition of wedges by an external heat flux, the problem treated in [8]-[10]. We also include a description of the ignition process of rectangular two-dimensional adiabatic bodies of finite size.

The rise in temperature in the solid is, at early times after application of the heat flux, given by the inert distribution, with negligible effects of the chemical reaction. These effects become significant when the maximum temperature, at the corner, reaches values close to a critical value $T_{I}$. Then, due to the strong sensitivity with temperature of the reaction rate, only a small increment in temperature, of order $R T_{I}^{2} / E$, is required to change the order of magnitude of the reaction rate, and cause a thermal runaway at a well-defined ignition time. We shall define the "ignition" temperature $T_{I}$ as the peak value of the inert temperature at the ignition time; for reasonable values of the reactivity of the solid, we may expect ignition to occur at values of $T_{I}$ such that $T_{I}-T_{0} \sim T_{0}$, where $T_{0}$ is the initial temperature.

After the heat flux $\tilde{q}$ is applied, the thermal wave heating the solid, of thermal conductivity $\lambda$, will reach a distance $\tilde{\delta}_{c}=\lambda T_{0} / \tilde{q}$ when the rise in surface temperature is of order $T_{0}$. When the solid size, $a$, is measured with $\tilde{\delta}_{c}$ we obtain one of the main parameters, $q=\tilde{q} a / \lambda T_{0}$, characterizing the ignition process. For values of $q \gg 1$, the ignition time is small compared with the heat conduction time across the solid, so that the thermal wave, with the higher temperature in its corners, is confined to a thin surface region of characteristic size $\tilde{\delta}_{c} \ll a$. The chemical reaction is confined to even smaller corner regions of size $\tilde{\delta}_{c} / \beta$, where $\beta=E / R T_{0}$ is the nondimensional activation energy. Therefore, the ignition process is determined by what happens very close to the corners, and the solid appears as semi-infinite when seen with the scale $\tilde{\delta}_{c}$.

The problem for a square corner will be considered in $\S 2$; while $\S 3$ is devoted to the analysis of ignition of wedges with arbitrary values of the tip angle. We shall treat in $\S 4$ the cases in which the heat flux is a nonconstant function of time. In the asymptotic analysis of $\S 2$ we will find that the asymptotic expansions involve powers of $1 / \ln \beta$, so that they are not of practical interest because of their slow convergence. The occurrence of logarithmic terms is closely related to the cylindrical geometry; due to the stronger geometrical effects, they will not be encountered in the analysis, given in the Appendix B, of the three-dimensional square corner.

In $\S 5$ we will give a description of the ignition process of cylindrical bodies, with adiabatic end surfaces so that the temperature field is two-dimensional. For the sake of brevity, we shall limit the analysis to bodies with rectangular cross-section and a constant heat flux. When $q$ is of order unity, or smaller but larger than $1 / \beta$, the size of the conduction region is of order $a$, but the chemical reaction is still confined to a small region around the corner. Therefore, the results of $\S 2$ are applicable with only minor changes involving quantities related to the inert temperature distribution. Finally, we shall analyze, in $\S \S 5.3$ and 5.4, the limiting cases $q \sim 1 / \beta$ and $q \ll 1 / \beta$; in these 
cases the reaction zone is no longer confined, during the ignition stage, to the corner region, but extends to the whole solid. When $q \ll 1$ the duration of the inert heating stage, proportional to $q^{-1}$, is large compared with the conduction time, and the spatial variations of temperature are of order $q$; on the other hand, the duration of the reactive stage is comparable to the conduction time if $q \sim 1 / \beta$, or larger when $q \ll 1 / \beta$. The case $q \sim 1 / \beta$ is a distinguished limiting case, for which, during the ignition stage, the chemical reaction effects must be taken into account together with the effects of the local heat accumulation and heat conduction; these last effects become dominant if $q \ll 1 / \beta$, when we encounter a nearly homogeneous explosion.

We shall consider, for simplicity in the presentation, that all the physical properties of the reactive solid, including its density $\rho_{s}$ and specific heat $c_{s}$, are constant and independent of the temperature. The chemical reaction will be modeled by an overall Arrhenius-type reaction rate. The effect of the reactant consumption will be neglected during the ignition period.

\section{The semi-infinite square corner.}

2.1. Formulation. Let us consider a two-dimensional semi-infinite reactive solid defined in cartesian coordinates $(\tilde{x}, \tilde{y})$ by $\tilde{x} \geq 0$ and $\tilde{y} \geq 0$. Initially the temperature of the solid is $T_{0}$, and starting at the instant $\tilde{t}=0$ a constant heat flux $\tilde{q}$, normal to the surface of the solid, is applied. We use $\tilde{\delta}_{c}=\lambda T_{0} / \tilde{q}$ and $\tilde{t}_{c}=\rho_{s} c_{s} \lambda T_{0}^{2} / \tilde{q}^{2}$ as characteristic length and time, respectively; and let $\varphi=T / T_{0}-1, B=\rho_{s} Q \tilde{B} \lambda T_{0} / \tilde{q}^{2}$ and $\beta=E / R T_{0}$ be dimensionless temperature, pre-exponential factor, and activation energy. $Q$ is the heat release per unit mass of fuel consumed in the reaction. The mathematical problem describing, for $t>0$, the reaction and heat conduction inside the solid can then be written as

$$
\begin{gathered}
\frac{\partial \varphi}{\partial t}=\frac{\partial^{2} \varphi}{\partial x^{2}}+\frac{\partial^{2} \varphi}{\partial y^{2}}+B e^{-\beta /(1+\varphi)} \\
\varphi_{y}(x, 0, t)+1=\varphi_{x}(0, y, t)+1=\varphi_{y}(x, \infty, t)=\varphi_{x}(\infty, y, t)=\varphi(x, y, 0)=0
\end{gathered}
$$

If we want to account for fuel consumption effects in the ignition process, we should include a factor $Y^{n}$ in the reaction term, where $Y$ is the reactant mass fraction and $n$ is the reaction order. Then, to describe the evolution of $Y$ we should add the equation $\partial Y / \partial t=-\gamma^{-1} B Y^{n} \exp (-\beta /(1+\varphi))$, together the initial condition $Y=1$. Here $\gamma=Q / c_{s} T_{0}$ is a nondimensional parameter characterizing the exothermicity of the reaction, typically large compared with unity. It is easy to see that the changes in $Y$ during the ignition stage are of order $1 / \gamma$, therefore small compared with one. Hence, if $\gamma \gg 1$ the fuel consumption may be safely neglected during this period.

In the applications the Zel'dovich number $\beta$, or nondimensional activation energy, is large compared with unity, typically of order 20; so that the Arrhenius exponential factor is very small compared with 1 . However, the Damköhler number $B$, or nondimensional frequency factor, is also very large compared with unity; so that, even if at the initial temperature the reaction term is negligible, when the local temperature reaches values close to a certain characteristic value the contribution of the reaction term to the local temperature rise will become of the same order, unity, as the rise due to the external heat flux.

In order to describe the structure of the solution for the typically large values of $\beta$ and $B$, we shall use the technique of large activation energy asymptotics, which implies the use of a double limit process. We begin by defining a value $\varphi_{c}$ of $\varphi$, typically of 
order 1 , for which the reaction term in (2.1) leads to rates of temperature rise of order unity. Namely, $\varphi_{c}$ will be defined by $B \exp \left(-\beta /\left(1+\varphi_{c}\right)\right)=\beta$; where the right-hand side takes into account the size, $1 / \beta$, of the reaction zone during the ignition stage, and the typical temperature changes, of order $1 / \beta$, in the same period. Equation (2.1) can be written in terms of $\varphi_{c}$ in the form

$$
\frac{\partial \varphi}{\partial t}=\Delta \varphi+\beta \exp \left[\frac{\beta}{1+\varphi_{c}} \frac{\varphi-\varphi_{c}}{1+\varphi}\right] .
$$

For large values of $\beta$, the reaction term in (2.3) will be exponentially small if $\varphi<\varphi_{c}$ and exponentially large if $\varphi>\varphi_{c}$. Thus, if in a given point $\varphi$ rises above $\varphi_{c}$ the temperature will rise steeply with $t$ towards infinity as a result of the infinite reaction rate. Therefore, in the limit $\beta \rightarrow \infty$ with $\varphi_{c}$ fixed, the solution of (2.2)-(2.3) blows up at a well-defined ignition time. The reactant consumption effects, not included in this analysis, introduce bounds to the maximum temperature, but they do not alter significantly either the time of thermal runaway or the temperature distribution when the peak value of $\varphi-\varphi_{c}$ is not large compared with $1 / \beta$.

Due to the initial condition, $\varphi=0$, it is clear that at early times, when $\varphi<\varphi_{c}$, (2.3) simplifies to the heat conduction equation, which when solved with the initial and boundary conditions (2.2) leads to the inert temperature distribution

$$
\varphi_{i}=2 \sqrt{t / \pi}\left(e^{-x^{2} / 4 t}+e^{-y^{2} / 4 t}\right)-(x \operatorname{erfc}(x / 2 \sqrt{t})+y \operatorname{erfc}(y / 2 \sqrt{t}))
$$

obtained by superposition of one-dimensional solutions. This expression shows that $\varphi_{i}$ is a monotonically increasing function of $t$ with its maximum value, $4 \sqrt{t / \pi}$, at the edge $(x=y=0)$. The inert stage ends when $\varphi_{i}$ approaches $\varphi_{c}$, and the reaction term ceases to be exponentially small to become of order unity in the small parameter $1 / \beta$. This will occur first at the edge, at a time close to $\pi \varphi_{c}^{2} / 16$, which can be considered as a first approximation to the ignition time. In order to show this, and to obtain a more precise value of the ignition time, we shall carry out in the following an asymptotic analysis of the problem (2.1)-(2.2) for large values of $\beta$. We begin, as in [2], by posing the problem of finding the value of $B$ that, for $\beta \gg 1$, leads to a thermal runaway, or exponentially large rate of temperature rise, at a given ignition time.

We select the ignition time to be $t=t_{I}=\mathcal{O}(1)$ and then we define $\varphi_{I}$ as the maximum inert temperature at $t=t_{I}$, i.e., $\varphi_{I}=\varphi_{i}\left(0,0, t_{I}\right)=4 \sqrt{t_{I} / \pi}$. For shortness in the notation, we also define a new parameter $\varepsilon$ as

$$
\varepsilon=\left(1+\varphi_{I}\right)^{2} / \beta \sqrt{\pi t_{I}},
$$

which is $\varepsilon=\mathcal{O}(1 / \beta) \ll 1$, since both $t_{I}$ and $\varphi_{I}$ are of order unity. We anticipate that for ignition to occur at $t=t_{I}=\pi \varphi_{I}^{2} / 16$, when $\varepsilon \ll 1$, B must be given by

$$
B=\frac{\beta}{\left(1+\varphi_{I}\right)^{2}} \exp \left(\frac{\beta}{1+\varphi_{I}}\right) \nu(\varepsilon) e^{b}
$$

with

$$
\nu(\varepsilon)=1 / \ln \varepsilon^{-1} .
$$

We shall replace, using (2.6), the unknown Damköhler number B in (2.1) by the unknown factor $e^{b}$, so that (2.1) takes the form

$$
\frac{\partial \varphi}{\partial t}=\Delta \varphi+\nu(\varepsilon) e^{b} \frac{\beta}{\left(1+\varphi_{I}\right)^{2}} \exp \left[\frac{\beta}{1+\varphi_{I}} \frac{\varphi-\varphi_{I}}{1+\varphi}\right] .
$$


The inert stage ends when the value of $\varphi_{i}$ at the edge of the corner differs from $\varphi_{I}$ by an amount of order $\varepsilon$. Then, during the short ignition stage due to the large activation energy, the effects of the chemical reaction become significant, even though the departures of $\varphi$ from $\varphi_{I}$, resulting from the reaction term and from the inert solution, are small. Expanding $\varphi_{i}$ about $t=t_{I}$ and $x=y=0$ we obtain

$$
\varphi_{i}(x, y, t)=\varphi_{I}+\frac{2\left(t-t_{I}\right)}{\sqrt{\pi t_{I}}}-(x+y)+o\left(\sqrt{x^{2}+y^{2}}, t-t_{I}\right)
$$

which dictates the appropriate time and space variables to describe the reaction zone in the ignition stage: $\sigma=2\left(t-t_{I}\right) \beta /\left(1+\varphi_{I}\right)^{2} \sqrt{\pi t_{I}}$ and $(\xi, \eta)=(x, y) \beta /\left(1+\varphi_{I}\right)^{2}$. In the variables $\sigma, \xi, \eta$, and $\psi=\left(\varphi-\varphi_{i}\right) \beta /\left(1+\varphi_{I}\right)^{2},(2.7)$ takes the form

$$
2 \varepsilon \frac{\partial \psi}{\partial \sigma}=\Delta \psi+\nu(\varepsilon) e^{b} \exp \left[\frac{\psi+\sigma-\xi-\eta+\varepsilon \varphi_{1}}{1+\frac{1+\varphi_{I}}{\beta}\left(\psi+\sigma-\xi-\eta+\varepsilon \varphi_{1}\right)}\right],
$$

where $\varphi_{1}$ is of order unity in the reaction region, in which the values of $\xi$ and $\eta$ are of order unity. Notice that the reduced Damköhler number $\nu(\varepsilon) e^{b}$ is to be obtained by requiring the thermal runaway to occur at $\sigma=0$.

2.2. The asymptotic analysis of the ignition stage. We shall carry out here an asymptotic analysis of the ignition stage for $\sigma \sim 1$ and large values of the activation energy, i.e., $\beta \gg 1$ or, equivalently, $\varepsilon \ll 1$. If the nonlinear effects of the heating due to the chemical reaction are to be important, $\psi$ must be of order unity during the ignition stage. Then, in first approximation, the transient term in (2.9) can be neglected, leading to the reactive-diffusive equation

$$
\Delta \psi+\nu(\varepsilon) \exp (\psi+\sigma+b-\xi-\eta)=0 .
$$

Although the reaction is confined to a region where $\rho=\sqrt{\xi^{2}+\eta^{2}}$ is of order unity, the heat generated

$$
\tilde{\omega}(\sigma)=\nu(\varepsilon) \omega(\sigma)=\nu(\varepsilon) \int_{0}^{\infty} \int_{0}^{\infty} \exp (\psi+\sigma+b-\xi-\eta) d \xi d \eta
$$

at $\sigma \sim 1$ has to be conducted to a much larger inert region, where $\rho$ is of order $1 / \sqrt{\varepsilon}$, to produce these values of $\psi$ of order unity. The spatial changes in temperature within the reaction zone required to generate this heat flux are, due to the cylindrical geometry, only moderately small compared with unity of order $\nu=-1 / \ln \varepsilon$; and this is then the order of the nondimensional reaction term.

After these scaling considerations, we can now take into account the fact that $\varepsilon \ll 1$ to look for an asymptotic description of the solution of $(2.9)$, together with the appropriate boundary and initial conditions, using an inner expansion for $\psi$ in the form $\psi=\psi_{0}(\xi, \eta, \sigma, \nu)+\varepsilon \psi_{1}(\xi, \eta, \sigma, \nu)+\cdots$ and an analogous outer expansion, in terms of $\sigma$ and the spatial variables $\sqrt{\varepsilon} \xi$ and $\sqrt{\varepsilon} \eta$; we shall also expand $b$ as $b_{0}(\nu)+$ $\varepsilon b_{1}(\nu)+\cdots$. Notice that, due to the matching conditions, the variables $\psi_{0}, \psi_{1}$, and $b_{0}$, $b_{1}$ in these expansions are, as it will be shown in the following, weakly dependent on $\varepsilon$, through $\nu(\varepsilon)$, which will be considered of order unity. The leading term of the inner expansion verifies $(2.10)$, the initial condition $\psi_{0}(\xi, \eta,-\infty, \nu)=0$, obtained from the matching condition with the inert stage, and the boundary conditions $\psi_{0, \xi}(0, \eta, \sigma, \nu)=$ $\psi_{0, \eta}(\xi, 0, \sigma, \nu)=0$, together with a boundary condition at $\rho \rightarrow \infty$, which must be deduced by matching with the inert region. 
For large values of $\rho$ the reaction term is exponentially small and, due to the absence of heat losses towards the walls, $\psi$ becomes independent of the angular coordinate $\theta$. Therefore, the nonzero heat flux $\tilde{\omega}(\sigma)$ at $\rho \rightarrow \infty$, given by (2.11) with $\psi$ and $b$ replaced by $\psi_{0}$ and $b_{0}$, leads to $\psi_{0} \sim-(2 / \pi) \tilde{\omega}(\sigma) \ln \rho+C(\sigma)$ as $\rho \rightarrow \infty$, where $C(\sigma)$ is a function of order unity satisfying $C(-\infty)=0$. As $\psi_{0}$ does not satisfy the condition $\psi_{0}(\infty, \theta, \sigma, \nu)=0$, we need to consider an outer transient-heat conduction zone in which the heat flux $\tilde{\omega}(\sigma)$ is accumulated. Using $r=\sqrt{2 \varepsilon} \rho$ as an outer space variable, (2.9) simplifies to a radial heat equation with a heat source of intensity $4 \tilde{\omega}(\sigma)$ located at the origin; the solution is given by $\psi=\pi^{-1} \int_{-\infty}^{\sigma} \tilde{\omega}(s)(\sigma-s)^{-1} \exp \left(-r^{2} / 4(\sigma-s)\right) d s$. This has, for $r \rightarrow 0$, the behavior $\psi \sim-(2 / \pi) \nu(\varepsilon) \omega(\sigma)(\ln r+\gamma-\ln 2)-\nu(\varepsilon) I(\sigma)+\mathcal{O}\left(r^{2}\right)$, where $\gamma$ is the Euler's constant and

$$
I(\sigma)=(1 / \pi) \int_{-\infty}^{\sigma}\left(\omega(\sigma) e^{-\sigma}-\omega(s) e^{-s}\right) e^{s}(\sigma-s)^{-1} d s .
$$

Matching between the inner and outer solutions leads to $\nu(\varepsilon)=1 / \ln \varepsilon^{-1}$, as anticipated in (2.6b), and $C(\sigma)=\omega(\sigma) / \pi-\nu(\varepsilon)((2 \gamma-\ln 2) \omega(\sigma) / \pi+I(\sigma))$. Then we can formulate the problem for $\psi_{0}$, as

$$
\begin{gathered}
\Delta \psi_{0}+\nu(\varepsilon) \exp \left(\psi_{0}+\sigma+b_{0}-\xi-\eta\right)=0 \\
\psi_{0}(\xi, \eta,-\infty, \nu)=\psi_{0, \xi}(0, \eta, \sigma, \nu)=\psi_{0, \eta}(\xi, 0, \sigma, \nu)=0 \\
\psi_{0}=\frac{1}{\pi} \omega(\sigma)-\nu(\varepsilon)\left(\frac{2}{\pi} \omega(\sigma)(\ln \rho+\gamma-\ln \sqrt{2})+I(\sigma)\right) \quad \text { as } \rho \rightarrow \infty
\end{gathered}
$$

with the functions $\omega(\sigma)$ and $I(\sigma)$ given by (2.11) and (2.12).

This nonlinear problem is to be solved numerically, for given values of $\nu(\varepsilon)$, marching in time since, even though (2.13) is not parabolic, the solution at a given instant is determined by the previous history through the function $I(\sigma)$.

When obtaining the problem $(2.13)-(2.14)$ we have considered $\nu(\varepsilon)$ to be of order unity. Since $\nu(\varepsilon) \rightarrow 0$ when $\varepsilon \rightarrow 0$, we can seek the solution of (2.13)-(2.14) in this limit in the form of Poincaré type expansions in powers of $\nu(\varepsilon)$ for $\psi_{0}(\xi, \eta, \sigma, \nu)=$ $\tilde{\psi}_{0}(\xi, \eta, \sigma)+\nu \tilde{\psi}_{1}(\xi, \eta, \sigma)+\cdots$ and $b_{0}(\nu)=\tilde{b}_{0}+\nu \tilde{b}_{1}+\cdots$. The leading term of $\psi_{0}$ is found to be $\tilde{\psi}_{0}=\tilde{\psi}_{0}(\sigma)$, so that the temperature in the reaction zone is spatially uniform in first approximation, and the boundary condition at $\rho \rightarrow \infty$ leads to the relation $\pi \tilde{\psi}_{0}=\exp \left(\tilde{\psi}_{0}+\sigma+\tilde{b}_{0}\right)$, giving $\tilde{\psi}_{0}$ as a function of $\sigma$. The derivative of $\tilde{\psi}_{0}, \tilde{\psi}_{0, \sigma}=\tilde{\psi}_{0} /\left(1-\tilde{\psi}_{0}\right)$, becomes unbounded at $\tilde{\psi}_{0}=1$, so that the existence of a thermal runaway at $\sigma=0$ requires $\tilde{b}_{0}=\ln (\pi / e)$. Therefore, the asymptotic analysis provides, for $\nu=1 / \ln \varepsilon^{-1} \ll 1$, the following relation between the ignition time and the pre-exponential factor

$$
B=B_{a}=\sqrt{\frac{\pi}{t_{I}}}\left(\varepsilon \ln \varepsilon^{-1}\right)^{-1} \exp \left(\frac{\beta}{1+\varphi_{I}}-1\right),
$$

which must be seen as the first term of an expansion of $B\left(t_{I}, \varepsilon\right)$ in powers of $\nu(\varepsilon)$. Notice that both $\varphi_{I}$ and $\varepsilon$ depend on $t_{I}$.

2.3. The numerical analysis of the ignition stage. The perturbation scheme of the last paragraph in $\S 2.2$ involves powers of $\nu(\varepsilon)=1 / \ln \varepsilon^{-1}$. Since, for the usual values of $\varepsilon, \nu(\varepsilon)$ is only moderately small, the asymptotic expansions for $\psi_{0}$ and $b_{0}$ in powers of $\nu$ can only be useful for unreasonably small values of $\varepsilon$. In addition, in the 
asymptotic description of $\S 2.2$ the increment in temperature evolves in a quasi-steady way; the analysis fails, obviously, for those values of $\sigma<0$ such that $\psi_{0, \sigma}=\mathcal{O}(1 / \varepsilon)$. Therefore, the ignition period includes the initial stage, described above, which ends with $\psi_{0, \sigma}$ growing to infinity with $\psi_{0}$ finite, and a shorter unsteady stage, in which the transient term should be retained in (2.13) and where the real runaway will be encountered. The analysis of the unsteady stage, requiring numerical computations, would provide a small correction to the value of $b_{0}$.

These considerations suggest that when solving (2.9) for small values of $\varepsilon$, with the aim of describing the ignition stage up to the time of the real thermal runaway, there is not great advantage in taking into account the existence of an inner reactivediffusive zone and an outer inert zone because the inner problem must be solved for various values of $\nu(\varepsilon)$, and the evaluation of $I(\sigma)$ forces us to use a time marching technique. Instead, we shall solve a simplified form of $(2.9)$, where because $\varepsilon \ll 1$ we neglect the $\mathcal{O}(\varepsilon)$ terms appearing in the Arrhenius exponent, but we shall retain the unsteady term in spite of the factor $\varepsilon$ to describe with a single equation the two region, two stage structure of the ignition period. We shall thus replace (2.9) by the nonlinear parabolic equation

$$
2 \varepsilon \psi_{0, \sigma}=\Delta \psi_{0}+\nu(\varepsilon) \exp \left(\psi_{0}+\sigma+b_{0}-\xi-\eta\right),
$$

whose solution for various small values of $\varepsilon$, with proper boundary and initial conditions, will provide the first term of a uniformly valid asymptotic expansion of $\psi$ in powers of $\varepsilon$. This is valid during the whole ignition stage and in the inner and outer zones. The solution of this problem must end in a thermal runaway at $\xi=\eta=\sigma=0$, and this condition determines the value $b(\varepsilon)$, with an error of order $\varepsilon$ due to the approximations made in the Arrhenius exponent.

In order to numerically solve (2.16) it is convenient to use polar coordinates and a translation in the time variable $\sigma$ to $\tau=\sigma+b_{0}+\ln \nu(\varepsilon)$; thus $\varepsilon$ is the only parameter left in (2.16). The initial and boundary conditions to be added to (2.16) are $\psi_{0}(\rho, \theta,-\infty)=\psi_{0, \theta}(\rho, 0, \tau)=\psi_{0, \theta}(\rho, \pi / 4, \tau)=\psi_{0}(\infty, \theta, \tau)=0$; in addition, $\psi_{0}$ must satisfy the regularity condition $\rho \psi_{0, \rho}=0$ at $\rho=0$. The resulting nonlinear problem was solved with the Newton-Kantorovich method, and a spatial discretization via a finite volume method. The Crank-Nicholson method was used, taking special care with the time steps to obtain quickly convergent solutions. Starting at $-\tau \gg 1$, the function $\psi_{0}$ is computed for increasing values of $\tau$, until $\psi_{0}$ and $\partial \psi_{0} / \partial \tau$ become sufficiently large. Then, the value $\tau_{I}$ of $\tau$ at ignition is obtained by insuring the behavior $\psi_{0}(0, \theta, \tau)=-\ln \left(e^{\tau_{I}}\left(\tau_{I}-\tau\right) / 2 \varepsilon\right)$ for small $\tau_{I}-\tau$ [13]. Ignition will take place at $t=t_{I}$, i.e., $\sigma=0$, only if $b_{0}$, characterizing the pre-exponential factor, takes the value $\tau_{I}(\varepsilon)-\ln \nu(\varepsilon)$. Then, recalling (2.6), $B$ as a function of $t_{I}$ and $\varepsilon$ is given by

$$
B=\left(\pi t_{I}\right)^{-1 / 2} \varepsilon^{-1} \exp \left(\tau_{I}(\varepsilon)+\frac{\beta}{1+\varphi_{I}}\right)
$$

If $B_{a}$ is the asymptotic value obtained in (2.15), it is easy to see that the ratio $B / B_{a}$ depends only on $\varepsilon$; so that (2.17) can be written in the form

$$
B\left(\varepsilon, t_{I}\right)=f(\varepsilon) B_{a}\left(\varepsilon, t_{I}\right)
$$

where $f(\varepsilon)=\pi^{-1} \ln \varepsilon^{-1} \exp \left(1+\tau_{I}(\varepsilon)\right)$ is a function of order unity. The functions $f(\varepsilon)$ and $\tau_{I}(\varepsilon)$ are shown in Fig. 1. 


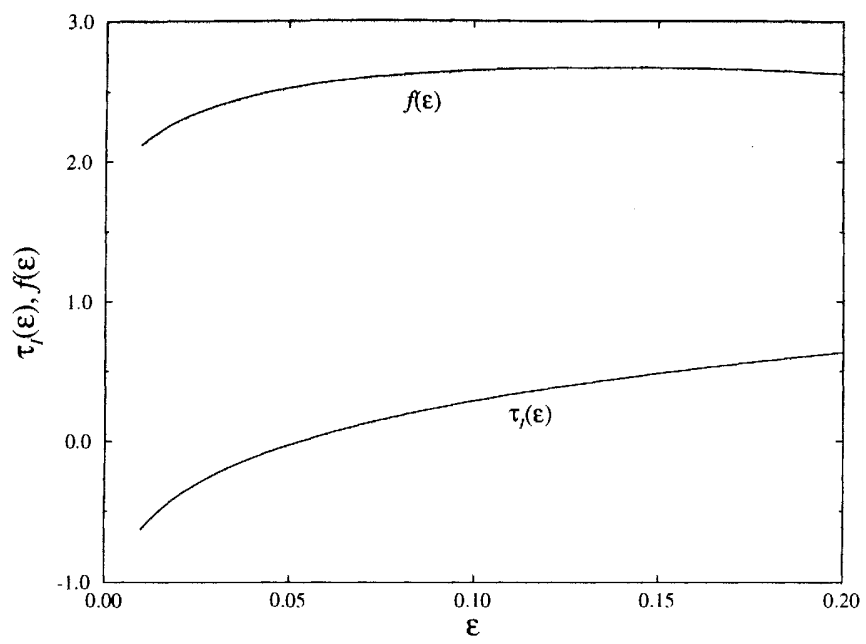

FIG. 1

There is perfect agreement of the results given by (2.17) with those found by Vorsteveld and Hermance [8], by numerical integration of the problem (2.1)-(2.2), including the reactant consumption. They did not previously perform any asymptotic analysis and, as a consequence, their equation for the temperature $\varphi$ contains two parameters: the activation energy and the pre-exponential factor, in addition to the fuel consumption parameter, $1 / \gamma$, which does not play a role in the time of thermal runaway because it is small compared with 1 .

3. The semi-infinite wedge. After analyzing the ignition of semi-infinite square corners, it is a simple matter to extend this analysis to other geometries. We have shown that, due to the large activation energy, the chemical reaction can be neglected during most of the ignition delay period, and then the temperature is given by the inert distribution. Only during a very short interval of time, proportional to the inverse of the activation energy, significant departures from the inert temperature occur. Therefore, the analysis of the ignition problem must begin by solving the inert nonsteady heat conduction equation for the given geometry under the external stimulus; from the inert solution we only need to look for the information required for the solution of the reactive stage. In this section we shall consider in some detail the problem of the ignition of a semiinfinite wedge with an angle $2 \alpha$ smaller than $\pi$. The ignition of wedges with angles larger than $\pi$ occurs away from the edge first, at the ignition time of the planar case.

By using polar coordinates and the notation of the beginning of $\S 2.1$, we obtain the equation (2.1), to be solved in the domain $[0, \infty[\times[0, \alpha]$ with the initial and boundary conditions $\varphi(r, \theta, 0)=\varphi_{\theta}(r, 0, t)=\varphi_{\theta}(r, \alpha, t)-r=\varphi_{r}(\infty, \theta, t)=0$. If ignition occurs at $t=t_{I}=\mathcal{O}(1)$, an expansion of the inert solution, $\varphi_{i}$, for $t-t_{I} \ll 1$ and $r \ll 1$ is required. For small distances from the origin compared with the depth of the layer heated by the thermal wave during the ignition period, i.e., for small values of $r / \sqrt{t_{I}}$, the following expansion of $\varphi_{i}$ is found (see Appendix A for details):

$$
\varphi_{i}(r, \theta, t)=\frac{\sqrt{\pi t_{I}}}{\alpha}+\frac{\sqrt{\pi}\left(t-t_{I}\right)}{2 \alpha \sqrt{t_{I}}}-r \frac{\cos \theta}{\sin \alpha}+\mathrm{o}\left(t-t_{I}, r\right),
$$


a generalization of (2.8) for arbitrary values of $\alpha<\pi / 2$. Notice that $\varphi_{I}\left(t_{I}\right)=\sqrt{\pi t_{I}} / \alpha$, $d \varphi_{I}\left(t_{I}\right) / d t=\sqrt{\pi} / 2 \alpha \sqrt{t_{I}}$ and $-\partial \varphi_{i}\left(0, \theta, t_{I}\right) / \partial r=\cos \theta / \sin \alpha$ increase with decreasing values of $\alpha$.

The reaction zone variables are defined as

$$
\sigma=\frac{\left(t-t_{I}\right) \beta}{2 \alpha\left(1+\varphi_{I}\right)^{2}} \sqrt{\frac{\pi}{t_{I}}}, \quad \rho=\frac{r \beta}{\sqrt{2} \sin \alpha\left(1+\varphi_{I}\right)^{2}}, \quad \psi=\frac{\left(\varphi-\varphi_{i}\right) \beta}{\left(1+\varphi_{I}\right)^{2}},
$$

so that order unity changes in $\sigma$ or $\rho$ imply a change of order unity in $\psi$. In these variables, and with $\varepsilon$ defined as in (2.5), the problem for the leading term of an expansion of $\psi$ in powers of $\varepsilon$ is found to be

$$
\begin{gathered}
\pi \varepsilon \frac{\sin ^{2} \alpha}{\alpha} \frac{\partial \psi}{\partial \tau}=\Delta \psi+\exp (\psi+\tau-\sqrt{2} \rho \cos \theta) \\
\psi(\rho, \theta,-\infty)=\psi_{\theta}(\rho, 0, \tau)=\psi_{\theta}(\rho, \alpha, \tau)=\psi(\infty, \theta, \tau)=0
\end{gathered}
$$

where

$$
\tau=\sigma+\ln \left(2 \sin ^{2} \alpha \frac{\left(1+\varphi_{I}\right)^{2}}{\beta} B \exp \left(-\frac{\beta}{1+\varphi_{I}}\right)\right) .
$$

The numerical solution shows a thermal runaway at a time $\tau=\tau_{I}(\varepsilon, \alpha)$. Therefore, the value of $B$, resulting in ignition of a wedge of angle $2 \alpha$ at $t=t_{I}$, is given by

$$
B=\frac{1}{2 \sin ^{2} \alpha} \frac{\beta}{\left(1+\varphi_{I}\right)^{2}} \exp \left(\tau_{I}+\frac{\beta}{1+\varphi_{I}}\right),
$$

where $\varphi_{I}=\sqrt{\pi t_{I}} / \alpha$. Figure 2 shows $\tau_{I}(\varepsilon, \alpha)$ as a function of $\varepsilon \sin ^{2} \alpha / \alpha$ for several values of $\alpha$.

For small values of $\alpha$, the time we have to wait to obtain $\varphi_{I}=\mathcal{O}(1)$ is $t_{I} \sim$ $\alpha^{2} \ll 1$, so that the parameter $\varepsilon$ in (3.3) can become of order unity, but then the factor $\varepsilon \sin ^{2} \alpha / \alpha \sim \varepsilon \alpha$ is still of order $1 / \beta$. In these cases the transverse conduction is so efficient that the inert temperature in the reaction zone becomes independent of $\theta$. Then, the heat production term is, in first approximation, independent of $\theta$. This fact, together the conditions $\psi(\rho, \theta,-\infty)=\psi_{\theta}(\rho, 0, \tau)=\psi_{\theta}(\rho, \alpha, \tau)=0$, implies $\psi=\psi(\rho, \tau)$, given by the one-dimensional problem

$$
\begin{gathered}
\pi \varepsilon \alpha \frac{\partial \psi}{\partial \tau}=\frac{1}{\rho} \frac{\partial}{\partial \rho}\left(\rho \frac{\partial \psi}{\partial \rho}\right)+\exp (\psi+\tau-\sqrt{2} \rho), \\
\left.\rho \psi_{\rho}(\rho, \tau)\right|_{\rho=0}=\psi(\rho,-\infty)=\psi(\infty, \tau)=0,
\end{gathered}
$$

obtained from (3.3)-(3.4) when the term $\partial^{2} \psi / \partial \theta^{2}$ is left out as result of the dominant role of the transverse conduction. This problem, containing $\varepsilon \alpha$ as the only parameter, is solved numerically for given small values of $\varepsilon \alpha$ to provide the function $\tau_{I}(\varepsilon, \alpha)=$ $\tau_{I}^{\prime}(\varepsilon \alpha)$, shown in Fig. 2. In terms of $\tau_{I}^{\prime}(\varepsilon \alpha)$ the value of $B$, such that ignition occurs at $t=t_{I}$, is given by the relation

$$
B=\frac{1}{2 \alpha^{2}} \frac{\beta}{\left(1+\varphi_{I}\right)^{2}} \exp \left(\tau_{I}^{\prime}+\frac{\beta}{1+\varphi_{I}}\right) .
$$

The problem (3.3)-(3.4) describes also the planar case for $\alpha=\pi / 2$. The corresponding function, $\tau_{I}(\varepsilon, \pi / 2)$, is also shown in Fig. 2. We have also plotted the value $\tau_{I}^{\prime \prime}(\varepsilon, \pi / 2)=\ln (2 \sqrt{\varepsilon})-0.431$, resulting from the asymptotic analysis of Liñán and Williams [2] for the planar case. 


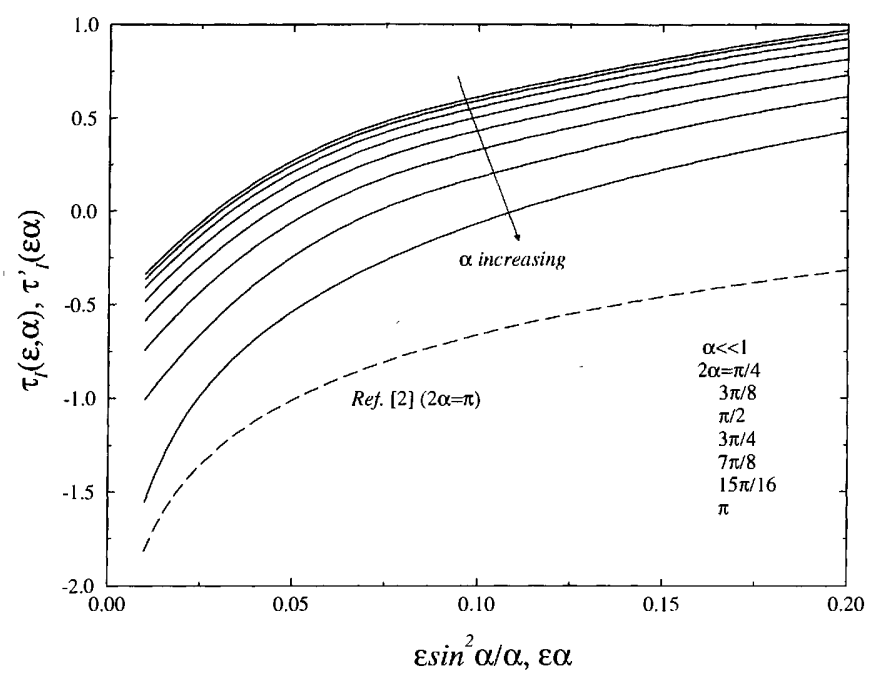

FIG. 2

\section{The ignition of a wedge subjected to an arbitrary heat flux.}

4.1. Equivalence with the constant heat flux case. The analysis of $\S \S 2$ and 3 , where a constant heat flux was considered, has shown that, when the nondimensional ignition time is $t_{I}=\mathcal{O}(1)$, the chemical reaction produces significant departures from the inert temperature, and then a thermal runaway, only during a short stage at the end of the ignition delay $t_{I}$. We can also expect the two stage character of the ignition process to be applicable to nonexotic cases of variable heat flux, $\tilde{q}(\tilde{t})$. The first inert stage will end only when the peak temperature is very close to an ignition temperature, determined by the kinetics of the reaction. For the description of the ignition processes, we must begin by analyzing the inert temperature history under the variable external input. The nondimensional peak temperature $\varphi_{I}(\tilde{t})=\left(\sqrt{\pi} / 2 \alpha T_{0} \sqrt{\rho_{s} c_{s} \lambda}\right) \int_{0}^{\tilde{t}} \tilde{q}(\tau)(\tilde{t}-\tau)^{-1 / 2} d \tau$, found at the edge of the wedge, will grow continuously in time if the derivative of the external heat flux $\tilde{q}(\tilde{t})$ stays always positive, but it will have a maximum at a certain time, $\tilde{t}=\tilde{t}_{m}$, if only a finite amount of energy is available for the heat flux. In this case if the reactivity of the solid, i.e., $\tilde{B}$, is sufficiently large ignition will occur before the peak temperature reaches its maximum value, at decreasing times for increasing values of the reactivity. On the other hand, if the reactivity is sufficiently small, i.e., for values of $\tilde{B}$ smaller than a certain critical value, ignition with a thermal runaway will not occur.

As in $\S 2$ the problem of describing the ignition under a variable heat flux involves finding the solution structure and the frequency factor of the reaction that, when the activation energy is large, leads to a thermal runaway at a given ignition time. We shall begin by defining a new characteristic time, $\tilde{t}_{c}$, as the time required by the heat flux to produce increments in surface temperature of order $T_{0}$, and a new characteristic length $\tilde{\delta}_{c}=\left(\lambda \tilde{t}_{c} / \rho_{s} c_{s}\right)^{1 / 2}$, as the depth, at $\tilde{t}=\tilde{t}_{c}$, of the layer heated by the heat flux. We can also use the additional order of magnitude relation $\lambda T_{0} / \delta_{c} \simeq \tilde{q}\left(\tilde{t}_{c}\right)=\tilde{q}_{c}$ to determine $\tilde{t}_{c}, \tilde{\delta}_{c}$, and $\tilde{q}_{c}$. We use $\tilde{q}_{c}$ to define the nondimensional pre-exponential factor and heat flux as $B=\rho_{s} Q \tilde{B} \lambda T_{0} / \tilde{q}\left(\tilde{t}_{c}\right)^{2}$ and $q=\tilde{q} / \tilde{q}\left(\tilde{t}_{c}\right)$. 
Let us assume that the ignition time is chosen to be $t_{I}=\mathcal{O}(1)$, and such that the inert temperature during the ignition stage can be represented by

$$
\varphi_{i}(r, \theta, t)=\varphi_{I}\left(t_{I}\right)+\dot{\varphi}_{I}\left(t_{I}\right)\left(t-t_{I}\right)-q_{I} r \frac{\cos \theta}{\sin \alpha}+\cdots,
$$

where

$$
\begin{aligned}
\varphi_{I}\left(t_{I}\right) & =\frac{\sqrt{\pi}}{2 \alpha} \int_{0}^{t_{I}} \frac{q(\tau)}{\sqrt{t_{I}-\tau}} d \tau, \\
\dot{\varphi}_{I}\left(t_{I}\right) & =\frac{\sqrt{\pi}}{2 \alpha}\left[\frac{q(0)}{\sqrt{t_{I}}}+\int_{0}^{t_{I}} \frac{d q(\tau)}{\sqrt{t_{I}-\tau}}\right], \quad q_{I}=q\left(t_{I}\right) .
\end{aligned}
$$

Since we want the chemical reaction to be frozen during the inert stage, and also far away from the reaction zone, the conditions $\dot{\varphi}_{I}\left(t_{I}\right)>0$ and $q_{I}>0$ must be fulfilled. Notice that $\dot{\varphi}_{I}\left(t_{I}\right)>0$ does not necessarily imply $d q\left(t_{I}\right) / d t>0$; so that the ignition can occur when the heat flux is decreasing. We can now use the analysis of $\S 3$ to calculate the increment in temperature due to the reaction during the ignition stage. Defining $\varepsilon$ as

$$
\varepsilon=\frac{2 \alpha \dot{\varphi}_{I}\left(1+\varphi_{I}\right)^{2}}{\pi q_{I}^{2} \beta}
$$

the numerical results of $\S 3$ allow us to obtain the following relation between the preexponential factor and the ignition time

$$
\rho_{s} Q \lambda T_{0} \tilde{B}=\frac{\tilde{q}_{I}^{2}}{2 \sin ^{2} \alpha} \frac{\beta}{\left(1+\varphi_{I}\right)^{2}} \exp \left(\tau_{I}(\varepsilon, \alpha)+\frac{\beta}{1+\varphi_{I}}\right),
$$

valid if $\dot{\varphi}_{I}\left(t_{I}\right)>0$ and $q_{I}>0$.

4.2. Critical conditions for ignition by a heat flux with a finite amount of energy. The previous analysis applies in particular when the conditions $q(t)>0$ and $d q(t) / d t>0$ hold for all values of $t$, obviously leading to a thermal runaway when the reactant consumption is neglected. However, ignition can take place under more general heat fluxes, for example, a short pulse with a finite amount of energy, i.e., $q(t)>0$ but $\int_{0}^{\infty} q(t) d t<\infty$. We can expect in this case that the inert temperature at the edge will reach a maximum value $\varphi_{m}$ at a time $t_{m}$. For large enough solid reactivities ignition will occur at times smaller than $t_{m}$ given by (4.4). However, for values of the frequency factor around a critical value, ignition will occur at times close to $t_{m}$ or it will not occur at all, as shown by Olmstead [5] and Lasseigne and Olmstead [7] in their analysis of the planar case. Similar critical conditions for ignition are found when analyzing the ignition of a gaseous reacting mixture with an instantaneous energy source [15], [16].

We shall consider here values of the frequency factor around the critical, such that the chemical reaction, although confined for large activation energies to the edge region of the wedge and times close to $t_{m}$, may lead to ignition for supercritical values of the frequency factor. For our ignition analysis the inert temperature distribution can be adequately described by

$$
\varphi_{i}(r, \theta, t)=\varphi_{m}+\frac{1}{2} \ddot{\varphi}_{m}\left(t-t_{m}\right)^{2}-q_{m} r \frac{\cos \theta}{\sin \alpha}+\cdots,
$$


where $\varphi_{m}=\varphi_{i}\left(0, \theta, t_{m}\right), \ddot{\varphi}_{m}=\partial^{2} \varphi_{i}\left(0, \theta, t_{m}\right) / \partial t^{2}<0$, and $q_{m}=q\left(t_{m}\right)>0$ are of order unity.

Using the expansion (4.5) for the inert temperature, it is a simple task to obtain, for the evolution of the increment in temperature due to the chemical reaction, the following problem:

$$
\begin{gathered}
\pi \varepsilon \frac{\sin ^{2} \alpha}{\alpha} \frac{\partial \psi}{\partial \sigma}=\Delta \psi+\lambda \exp \left(\psi-\sigma^{2}-\sqrt{2} \rho \cos \theta\right) \\
\psi(\rho, \theta,-\infty)=\psi_{\theta}(\rho, 0, \sigma)=\psi_{\theta}(\rho, \alpha, \sigma)=\psi(\infty, \theta, \sigma)=0
\end{gathered}
$$

where

$$
\begin{aligned}
\varepsilon=\sqrt{2\left|\ddot{\varphi}_{m}\right|} \frac{\alpha\left(1+\varphi_{m}\right)^{3}}{\pi q_{m}^{2} \beta \sqrt{\beta}}, & \lambda=2 \sin ^{2} \alpha \frac{\left(1+\varphi_{m}\right)^{2}}{\beta} e^{-\frac{\beta}{1+\varphi_{m}} \frac{\rho_{s} \dot{Q} \tilde{B} \lambda T_{0}}{\tilde{q}_{m}^{2}}} \\
\sigma=\left(t-t_{m}\right) \sqrt{\frac{\left|\ddot{\varphi}_{m}\right|}{2}} \frac{\sqrt{\beta}}{\left(1+\varphi_{m}\right)}, & \rho=r \frac{q_{m}}{\sqrt{2} \sin \alpha} \frac{\beta}{\left(1+\varphi_{m}\right)^{2}}
\end{aligned}
$$

and $\psi$ is defined as in (3.2).

The results of the numerical solution of (4.6)-(4.7) show blow up at an ignition time $\sigma_{I}=\sigma_{I}(\lambda, \varepsilon, \alpha)$, only if $\lambda>\lambda_{*}(\varepsilon, \alpha)$. Figure 3 shows the function $\lambda_{*}(\varepsilon, \alpha)$ as a function of $\varepsilon \sin ^{2} \alpha / \alpha$, and Fig. 4 shows the ignition time $\sigma_{I}$ as a function of $\lambda$ and several values of $\varepsilon$. Hence for given values of $\lambda$, $\alpha$ and $\varepsilon$, with $\lambda>\lambda_{*}(\varepsilon, \alpha)$, ignition takes place at $t_{I}=t_{m}+\sigma_{I} \sqrt{2\left(1+\varphi_{m}\right)^{2} /\left|\ddot{\varphi}_{m}\right| \beta}$. Notice that, since the function $\lambda_{*}(\varepsilon, \alpha)$ is monotonically increasing with $\varepsilon$, for each value of $\lambda$ there exists a maximum value of $\varepsilon, \varepsilon_{*}(\lambda, \alpha)$, such that ignition will occur only if $\varepsilon<\varepsilon_{*}(\lambda, \alpha)$. For $\alpha \ll 1$ and $\alpha=\pi / 2$ the problem (4.6)-(4.7) becomes, as in $\S 3$, one-dimensional. The numerical solution for the limiting case $\alpha=\pi / 2$ can be compared with the result, $\lambda_{*}^{\prime}(\varepsilon) \simeq 0.9 \sqrt{\varepsilon}$, from the asymptotic analysis of Lasseigne and Olmstead [7] for the one-dimensional case, also shown in Fig. 3.

\section{Ignition of cylindrical rectangular solids.}

5.1. Formulation. We have analyzed in $\S 2$ the ignition process in a semi-infinite square corner, and now we shall consider the ignition of cylindrical solids of rectangular cross-section. We assume that their length is infinite, or finite with adiabatic end crosssections, so that the temperature field is two-dimensional. The following analysis will show the existence of three different regimes, depending on the order of magnitude of the external heat flux.

Let us consider a reactive body of rectangular shape with dimensions $2 a$ and $2 b$ $(b \geq a)$. Starting at $\tilde{t}=0$ a constant external heat flux, $\tilde{q}$, is applied. The nondimensional problem that describes the evolution of the nondimensional temperature, $\varphi=\left(T-T_{0}\right) / T_{0}$, in terms of the spatial coordinates, measured with the shorter dimension $a$, and the time, measured with the conduction time, $\tilde{t}_{c}=\rho_{s} c_{s} a^{2} / \lambda$, is

$$
\begin{gathered}
\frac{\partial \varphi}{\partial t}=\Delta \varphi+B \exp \left[-\frac{\beta}{1+\varphi}\right] \quad \text { in } \Omega=[0,1] \times[0, l], \\
\varphi_{x}(0, y, t)=\varphi_{y}(x, 0, t)=\varphi_{x}(1, y, t)-q=\varphi_{y}(x, l, t)-q=\varphi(x, y, 0)=0 .
\end{gathered}
$$




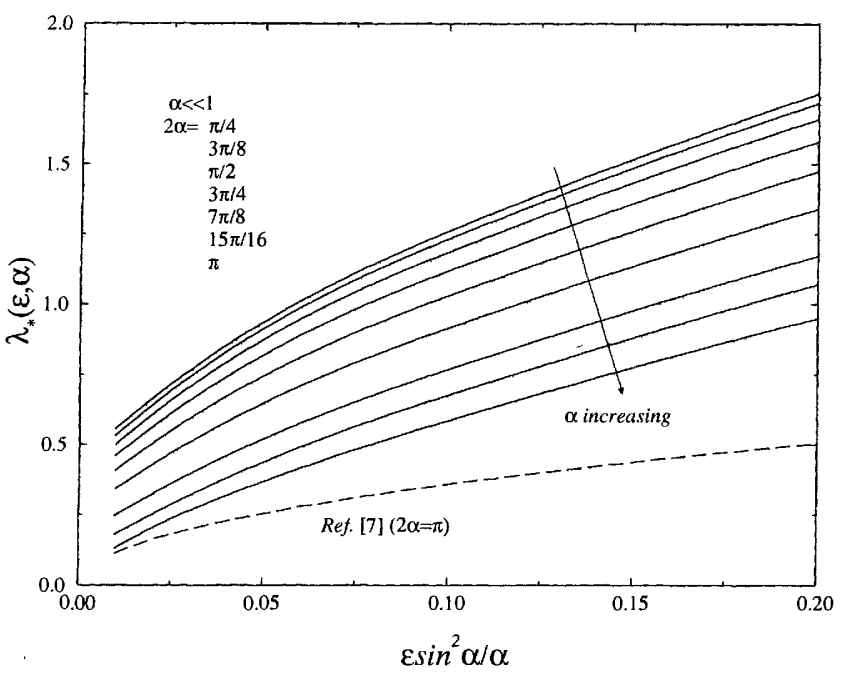

FIG. 3

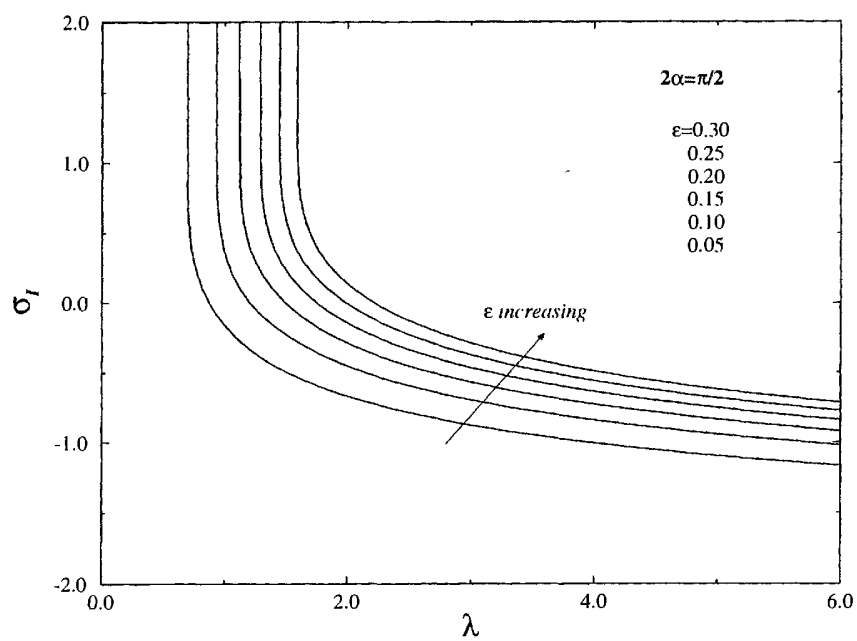

FIG. 4

Here, $q=\tilde{q} a / \lambda T_{0}$ and $B=\rho_{s} Q \tilde{B} a^{2} / \lambda T_{0}$ are the nondimensional heat flux and preexponential factor, and $l=b / a \geq 1$ is the slenderness of the cross-section.

We shall deal with cases for which at early times $B \exp (-\beta /(1+\varphi))$ is exponentially small, so that the chemical reaction is frozen. The temperature is then given by the inert distribution, which can be written as $\varphi_{i}=q F(x, y, t)$, where $F$ is the inert solution corresponding to $q \equiv 1$. The function $F$ is well described for $t \ll 1$ by

$$
F(x, y, t)=2 \sqrt{t}\left(\operatorname{ierfc}\left(\frac{1-x}{2 \sqrt{t}}\right)+\operatorname{ierfc}\left(\frac{l-y}{2 \sqrt{t}}\right)\right)
$$

where $\operatorname{ierfc}(z)=\int_{z}^{\infty} \operatorname{erfc}(t) d t=e^{-z^{2}} / \sqrt{\pi}-z \operatorname{erfc}(z)$. Whereas for $t=\mathcal{O}(1)$ we shall 
use

$$
\begin{aligned}
F(x, y, t)= & t\left(1+\frac{1}{l}\right)+\frac{1}{2}\left(x^{2}+\frac{1}{l} y^{2}\right)-\frac{1}{6}(1+l) \\
& -\frac{2}{\pi^{2}} \sum_{n=1}^{\infty} \frac{(-1)^{n}}{n^{2}}\left(e^{-n^{2} \pi^{2} t} \cos n \pi x+l e^{-n^{2} \pi^{2} t / l^{2}} \cos n \pi \frac{y}{l}\right) .
\end{aligned}
$$

Notice that the maximum value of the inert temperature is attained at the corners and grows as $4 q \sqrt{t / \pi}$ for $t \ll 1$, and as $q t\left(1+l^{-1}\right)+q(1+l) / 3$ for $t \sim 1$.

The chemical reaction comes into play after a time $t_{I}$, such that the corresponding maximum temperature, $\varphi_{I}$, is such that the factor $B \exp \left(-\beta /\left(1+\varphi_{I}\right)\right)$ is at least of algebraic order in the small parameter $\beta^{-1}$. As in the previous sections, the reaction term must only be taken into account where $\varphi-\varphi_{I} \sim\left(1+\varphi_{I}\right)^{2} / \beta$, and then it is convenient to use as dependent variable $\psi=\left(\varphi-\varphi_{i}\right) \beta /\left(1+\varphi_{I}\right)^{2}$, of order unity during the ignition transient; the reaction zone is located where $\varphi-\varphi_{I}=\mathcal{O}(1 / \beta)$. Next we have to determine the time and space scales associates with the ignition stage, and we shall find that they depend on the order of magnitude of $q$.

5.1.1. Case A: $\boldsymbol{q} \gg$ 1. For very large values of $q$, the time we must wait to obtain $\varphi_{I}=\mathcal{O}(1)$ is given by $t_{I} \sim 1 / q^{2} \ll 1$, with the thickness of the conduction layer $\delta_{c} \sim 1 / q \ll 1$. The time scale associated with the ignition stage is $\sim 1 / \beta q^{2}$, determined by $\varphi_{i}-\varphi_{I} \sim q\left(t-t_{I}\right) / \sqrt{t_{I}} \sim 1 / \beta$; the heat of the reaction is conducted to a zone of thickness $\delta_{r} \sim 1 / q \sqrt{\beta} \ll \delta_{c}$. Hence, for $q \gg 1$ both the layer heated by the external heat flux and the sublayer heated by the effect of the chemical reaction are very thin; so that the solid appears as a semi-infinite one, and the results of $\S \S 2$ and 3 are directly applicable.

5.1.2. Case B: $\boldsymbol{q} \sim \mathbf{1}$. When order unity values of $q$ are considered, the time $t_{I}$ becomes also of order unity and is given by $t_{I} \sim 1 / q=\mathcal{O}(1)$, which implies $\delta_{c}=\mathcal{O}(1)$. The time scale for the reaction stage is $1 / q \beta \ll 1$, and the spatial scale for the heated sublayer is $\delta_{r} \sim 1 / \sqrt{q \beta} \ll 1$. This means that, although the thermal wave due to the external flux has reached the core of the solid, the reaction layer and the layer receiving the heat of the reaction are thin compared with the size of the solid; so that with these scales the solid appears as semi-infinite. Therefore, we must only modify the inert distribution, which is no longer that corresponding to the infinite solid, to take into account the effect of the finite size.

5.1.3. Case C: $\boldsymbol{q} \ll \mathbf{1}$. Finally, for small values of $q$ we obtain $t_{I} \sim 1 / q \gg 1$, while the spatial variations of $\varphi_{i}$ are of order $q$. The duration of the ignition stage is $\sim 1 / q \beta$, so that we encounter a distinguished regime (Case C.2) when $q \beta \sim 1$, with the reaction zone extending to the whole solid, with two subregimes for $1 \gg q \gg 1 / \beta$ (Case C.1) and $q \ll 1 / \beta$ (Case C.3).

Notice that for these small values of $q$, the ignition delay time, or the time required for $\varphi_{i}$ to grow up to values of order unity, is larger than the conduction time; so that the temperature becomes spatially nearly uniform with variations of order $q$. When $q \ll 1$, the inert temperature during the ignition period is given by

$$
\varphi_{i}(x, y, t)=q t\left(1+l^{-1}\right)+\frac{q}{2}\left(x^{2}+l^{-1} y^{2}\right)-\frac{q}{6}(1+l)+\text { e.s.t. }
$$

5.2. Analysis of the limiting cases $q \gg 1 / \beta$ (Cases A, B y C.1). For all values of $q$ such that $q \beta \gg 1$, i.e., Cases A, B y C.1, both the thickness of the reaction 
zone and that of the layer receiving the heat of the reaction during the ignition period are small compared with the body size. In Cases B and C.1 the thermal wave reaches the core of the solid before ignition takes place, while in Case A the conduction layer is still thin. Therefore, in these three cases the solid appears as semi-infinite when seen from the reaction zone during the ignition stage; then, the description of the ignition process for these cases is the same, with simplifications in the inert temperature distribution for the extreme cases.

Because the reaction zone will be located near the point $(1, l)$ and the ignition event will take place around $t=t_{I}$, the inert temperature may be approximated by

$$
\varphi_{i}(x, y, t)=\varphi_{I}+\dot{\varphi}_{I}\left(t-t_{I}\right)-q(1-x)-q(l-y)+o\left(t-t_{I}, 1-x, l-y\right),
$$

where $\varphi_{I}=\varphi_{i}\left(1, l, t_{I}\right)$ and $\dot{\varphi}_{I}=\partial \varphi_{i}\left(1, l, t_{I}\right) / \partial t$ must be computed from the inert solution (5.4) or the asymptotics forms (5.3) or (5.5). Notice that for $t_{I} \ll 1$ (case A), the expressions for $\varphi_{I}$ and $\dot{\varphi}_{I}$ are those obtained in $\S 2$, when considering the semi-infinite square corner.

The expansion (5.6) dictates the following variables for the reaction zone

$$
\begin{aligned}
\tau & =\frac{\beta \dot{\varphi}_{I}}{\left(1+\varphi_{I}\right)^{2}}\left(t-t_{I}\right)+\ln \left(\frac{\left(1+\varphi_{I}\right)^{2}}{\beta q^{2}} B \exp \left(-\frac{\beta}{1+\varphi_{I}}\right)\right), \\
(\xi, \eta) & =\frac{\beta q}{\left(1+\varphi_{I}\right)^{2}}(1-x, l-y) .
\end{aligned}
$$

Introducing (5.6), (5.7) and the definition of $\psi$, in (5.1)-(5.2), we obtain, as in $\S 2.3$, the following problem for $\psi$ :

$$
\begin{gathered}
2 \varepsilon \frac{\partial \psi}{\partial \tau}=\Delta \psi+\exp (\psi+\tau-\xi-\eta) \\
\psi(\xi, \eta,-\infty)=\psi_{\xi}(0, \eta, \tau)=\psi_{\eta}(\xi, 0, \tau)=\psi\left(\xi^{2}+\eta^{2} \rightarrow \infty, \tau\right)=0
\end{gathered}
$$

where $\varepsilon=\dot{\varphi}_{I}\left(1+\varphi_{I}\right)^{2} / 2 q^{2} \beta \ll 1$. For $\alpha=\pi / 2$ this problem is identical to (3.3)-(3.4). The solution blows up at the time $\tau=\tau_{I}(\varepsilon)$ obtained in $\S 2.3$. Therefore, if we want the ignition time to be $t_{I}$, the frequency factor $\tilde{B}$ is given by

$$
\frac{\rho_{s} Q \tilde{B} a^{2}}{\lambda T_{0}}=\frac{q^{2} \beta}{\left(1+\varphi_{I}\right)^{2}} \exp \left(\tau_{I}(\varepsilon)+\frac{\beta}{\left(1+\varphi_{I}\right)}\right),
$$

valid for all values of $q$ such that $q \beta \gg 1$. Notice that the slenderness, $l$, appears only in the definition of $\varphi_{I}$ and $\dot{\varphi}_{I}$ in the Cases B and C.1, but not in the reaction zone problem (5.8)-(5.9).

5.3. Analysis of the distinguished limit $q \sim 1 / \beta$ (Case C.2). When $q \sim$ $1 / \beta$ we can divide the ignition process into two stages: an inert heating stage, of duration proportional to $\beta$, and a reacting stage, with a duration of order unity, the order of the conduction time across the body. During the first stage the temperature becomes spatially nearly uniform, with spatial variations of order $q$, which are of the order, $1 / \beta$, of the variations in temperature encountered in the ignition stage. Therefore, the reaction zone extends to the whole solid during this stage, with all the terms in the conservation equation of the same order. 
If we define $\Gamma=q \beta /\left(1+\varphi_{I}\right)^{2}=\mathcal{O}(1)$, from (5.5) we can write the inert temperature during the ignition stage in the form

$$
\varphi_{i}=\varphi_{I}+\Gamma \frac{\left(1+\varphi_{I}\right)^{2}}{\beta}\left(\left(1+\frac{1}{l}\right)\left(t-t_{I}\right)-\frac{1}{2}\left(1-x^{2}\right)-\frac{1}{2 l}\left(l^{2}-\eta^{2}\right)\right),
$$

where $\varphi_{I} / q=t_{I}\left(1+l^{-1}\right)+(1+l) / 3$. Then we introduce the new time variable

$$
\tilde{\tau}=\Gamma\left(1+\frac{1}{l}\right)\left(t-t_{I}\right)+\ln \left(\frac{\beta}{\left(1+\varphi_{I}\right)^{2}} B \exp \left(-\frac{\beta}{1+\varphi_{I}}\right)\right),
$$

and, after neglecting terms of order $1 / \beta$ or smaller in the conservation equation for $\psi$, we obtain the following problem for the lowest-order approximation for $\psi$ in $\Omega=$ $[0,1] \times[0, l]$

$$
\begin{gathered}
\Gamma\left(1+\frac{1}{l}\right) \frac{\partial \psi}{\partial \tilde{\tau}}=\Delta \psi+\exp \left[\psi+\tilde{\tau}-\frac{\Gamma}{2}\left(\left(1-x^{2}\right)+\frac{1}{l}\left(l^{2}-y^{2}\right)\right)\right] \\
\psi(x, y,-\infty)=0 \quad \text { in } \Omega, \quad \frac{\partial \psi(x, y, \tilde{\tau})}{\partial n}=0 \quad \text { in } \partial \Omega
\end{gathered}
$$

Notice that we use the initial condition at $\tilde{\tau}=-\infty$ because the duration of the reaction stage is of order unity while the duration of the inert heating stage is of order $\beta \gg 1$.

The numerical solution of (5.13)-(5.14) blows up at $\tilde{\tau}=\tilde{\tau}_{I}(\Gamma, l)$, shown in Fig. 5 . If the ignition time is $t=t_{I}$, the relation

$$
\frac{\rho_{s} Q \tilde{B} a^{2}}{\lambda T_{0}}=\frac{q^{2}\left(1+\varphi_{I}\right)^{2}}{\beta} \exp \left(\tilde{\tau}_{I}(\Gamma, l)+\frac{\beta}{1+\varphi_{I}}\right)
$$

determines $\tilde{B}$.

For large values of the slenderness, $l$, the inert distribution (5.3) or (5.4) must be replaced by its appropriate representation for $l=\infty$, leading to the equation $\Gamma \partial \psi / \partial \tilde{\tau}=\Delta \psi+\exp \left(\psi+\tilde{\tau}-\Gamma\left(\left(1-x^{2}\right) / 2-y\right)\right)$ to be solved in $\Omega=[0,1] \times[0, \infty[$ with the initial and boundary conditions given in (5.14). The solution blows up at $\tilde{\tau}=\tilde{\tau}_{I}(\Gamma, \infty)$, also represented in Fig. 5 .

When solving (5.13)-(5.14) for $\Gamma \gg 1$, we notice that the reaction zone is confined to the small corner region, so that the results of $\S 2.3$ can be used to obtain $\tilde{\tau}_{I}(\Gamma, l)=$ $\tau_{I}((l+1) / 2 l \Gamma)+2 \ln \Gamma$. The solution for $\Gamma \ll 1$ is given in the following $\S 5.4$.

5.4. Analysis of the limit $q \gg 1 / \beta$ (Case C.3). When $\Gamma \sim q \beta \ll 1$, the time to obtain $\varphi_{I} \sim 1$ is $t_{I} \sim 1 / q \gg \beta$ and the duration of the reactive stage is $\mathcal{O}(1 / \Gamma) \gg 1$. Due to the dominant effect of heat conduction the spatial variations of temperature resulting from the external heating, of order $q$, are much smaller than the increments in temperature introduced by the chemical reaction, of order $1 / \beta \gg q$. Therefore, during the ignition stage, the inert temperature is, in the first approximation, spatially uniform and can be represented by $\varphi_{i}=q\left(1+l^{-1}\right) t$, which determines the same reaction rate at each point of the solid. This, together with the boundary condition $\partial \psi /\left.\partial n\right|_{\partial \Omega}=0$, allows us to neglect the spatial variations of $\psi$. Hence, if $q \ll 1 / \beta$ the ignition event is the result of a nearly adiabatic homogeneous explosion, which is described by (5.13) when the diffusive term is left out and $\Gamma$ is replaced by zero in the exponent of the reaction term, plus the initial condition of (5.14). The solution of this initial value problem is $e^{\tilde{\tau}}=\Gamma(1+1 / l)\left(1-e^{-\psi}\right)$, which leads to the 


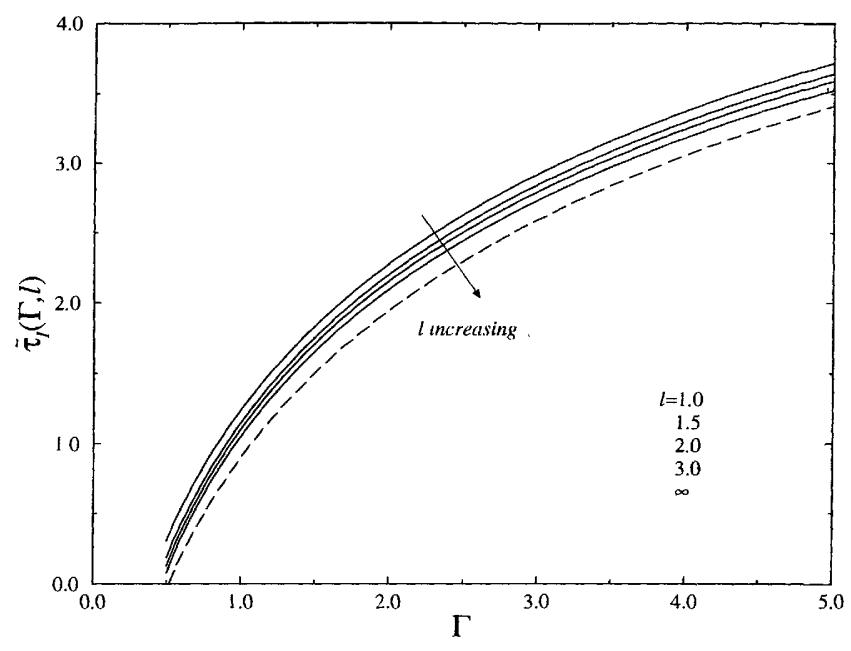

FIG. 5

asymptotic representation $\tilde{\tau}_{I}=\ln (\Gamma(1+1 / l))$ of the function $\tilde{\tau}_{I}(\Gamma, l)$ for small values of $\Gamma$. Therefore, if the ignition time is $t=t_{I}$, the pre-exponential factor is given by

$$
\frac{\rho_{s} Q \tilde{B} a^{2}}{\lambda T_{0}}=q\left(1+\frac{1}{l}\right) \exp \left(\frac{\beta}{1+\varphi_{I}}\right) .
$$

6. Concluding remarks. We have shown how a chemical reaction of large activation energy can be triggered to occur in a short time only when the peak temperature of the reacting body has been raised, during an inert heating stage, from its initial value $T_{0}$ to an "ignition" value $T_{I}$. Then, the incipient effects of the chemical reaction, although confined to the region where $T-T_{I}$ is of the order of the Frank-Kamenetskii temperature $R T_{I}^{2} / E$, will produce at the end of a short ignition stage a local thermal runaway. This will originate (see the analysis of Kapila [4]) a fast deflagration wave that propagates across the body to complete the chemical reaction.

We have seen how the evolution of the temperature distribution within a body, of size $a$ and heat conductivity $\lambda$, depends on the nondimensional value $q=\tilde{q} a / \lambda T_{0}$ of the external heat flux. For values of $q$ of order unity the time required for the inert temperature to reach the ignition temperature is of the order of the heat conduction time across the body. But for values of $q \gg 1$ the thermal heating during the inert stage will be confined to a thin surface layer, with identical temperature history along the surface of the body if this is smooth; in this case the ignition time will be equal to that of a planar semi-infinite body [1], [2]. However, if the surface has edges the temperature will rise more rapidly in these edges and the ignition time will be shortened. Thus, for a rectangular-shaped body subjected to a large external heat flux, $q \gg 1$, the reaction will be confined during the ignition stage to small regions close to the corners, and the ignition time will be independent of the body size.

When the external heating rate is small, $q \ll 1$, the temperature rises slowly with time, and shows small spatial variations of order $q T_{0}$. When these are of the order of $R T_{I}^{2} / E$, they must be taken into account when describing the structure of the ignition stage; although they may be neglected for values of $q \ll R T_{I} / E$.

The ignition time is given, for large values of the nondimensional activation energy, $\beta=R T_{0} / E \gg 1$, by the time required to reach the ignition temperature $T_{I}$. This 
temperature is given, with errors of order $R T_{I}^{2} / E$, by the order of magnitude estimate

$$
B \exp \left(-E / R T_{I}\right)=E / R T_{0},
$$

so that if we want to calculate a first approximation for the ignition time we could be dispensed of analyzing the short ignition stage that precedes the thermal runaway. Or in other words, the value of the nondimensional frequency factor $B$ leading to an ignition time $t_{I}$, or ignition temperature $T_{I}$, is given in order of magnitude by the above relation (6.1). The precise value of $B$ can only be determined, as done in the previous sections, after the analysis of the ignition stage. Notice that the value of $T_{I}$ is mainly determined by the kinetics, but the time, $t_{I}$, to reach this value is determined, through the inert distribution by the geometry of the solid.

The structure of the ignition stage for bodies with wedge type edges was analyzed in $\S \S 2$ and 3 for values of $q \gtrsim 1$ when the reaction zone is confined to these wedge type regions. Although during the heating stage the time derivative term is smaller, by a factor $1 / \beta$, than the heat conduction and reaction terms in the reaction zone, the temperature level can only be determined by taking into account how the heat generated by the reaction is conducted to an outer inert region of larger size by a factor $\sqrt{\beta}$. The temperature distribution during the ignition stage is given by the value of $\psi$, solution of the problem (3.3)-(3.4), with errors of order $1 / \beta$ in $\psi$. If we want to use inner and outer expansions of the Poincare type to solve this problem, we find that, due to the $\ln r$ terms appearing in the solution of the heat conduction equation, the expansion parameters are integer powers of $\nu \sim 1 / \ln \beta$; then, accurate results can only be obtained for unrealistic large values of $\beta$. Thus, we were forced to solve numerically the problem (3.3)-(3.4) for small values of $\varepsilon$ in order to obtain an accurate relation between the frequency factor and the ignition time. However, the method of inner and outer expansions can be used for the description of the ignition stage of three-dimensional square corners, as shown in Appendix B, using expansions in the small parameter $1 / \sqrt{\beta}$.

While the ignition time for large values of the nondimensional flux $q$ is independent of the body size and shape, with the thermal runaway at the edges, this is not the case when $q$ is of order or smaller than unity. We have carried out the analysis for the inert and ignition stages for cylindrical bodies of rectangular cross sections and various values of the slenderness $l$. The results for $l=\infty$ can be used to describe the ignition of thin, coin-shaped plates (bounded by a smooth, straight, cylindrical surface), if the ratio of their thickness $2 a$ to the characteristic dimension $L$ of the surface of the plate is small. The effect of the end sections can be seen when one compares the ignition times of the semi-infinite slab $(l=\infty)$ and the infinite slab without edges. Figure 6 shows the nondimensional pre-exponential factor $\rho_{s} Q \tilde{B} a^{2} / \lambda T_{0}$ as a function of the groupings $q \sqrt{t_{I}}$ and $q t_{I}$, characterizing, for $q \gg 1$ and $q \sim 1 / \beta$, respectively, the maximum inert temperature at the ignition time. Notice the strong reduction in the ignition time if $q \gg 1$, for a given value of the nondimensional pre-exponential factor, due to the fact the ignition process is controlled by the corner effect. In the opposite case, $q \sim 1 / \beta$, the temperature is nearly uniform and the reduction is much smaller.

We found regimes similar to those described in this paper in our analysis of the ignition of a rectangular solid, with Dirichlet type conditions resulting from a step in surface temperature [11]. In that case we found a critical value of the Damköhler number, such that no ignition occurred for Damköhler numbers less than the critical one. Since in the present mathematical model we have Neumann type boundary conditions, with predetermined heat flux to the solid from the surrounded external medium, the 


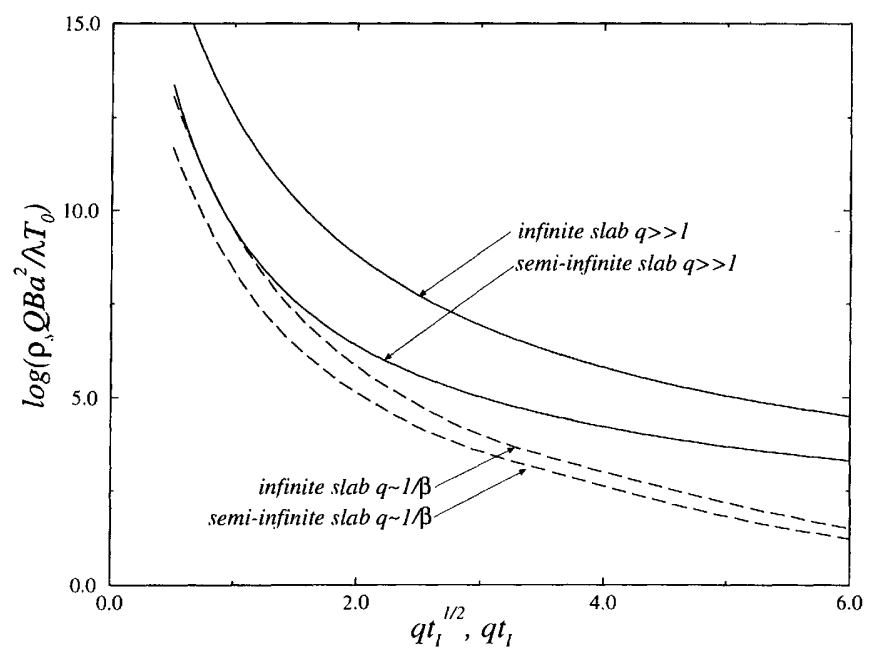

FIG. 6

minimum temperature in the solid is continuously increasing, so that self-ignition will always take place, even when the external heat flux disappears. However, the time delay to the thermal runaway can be so long, even at the new solid temperature, that the heat losses to the surrounded medium cannot be neglected.

We have not included in this work the effect of the reactant consumption, under the assumption that the heat-release parameter $\gamma$ is sufficiently large. When this effect is taken into account if $\gamma$ is of order unity, one may expect to encounter a direct transition to a deflagration wave, without thermal runaway, as a result of the heating from an external heat flux.

Appendix A. The inert solution for a wedge of angle $2 \alpha$. Let us consider the problem

$$
\begin{gathered}
\left.\frac{\partial \varphi}{\partial t}=\Delta \varphi \quad \text { in } \Omega=\right] 0, \infty[\times] 0, \alpha[, \quad t>0, \\
\varphi_{\theta}(r, 0, t)=0, \varphi_{\theta}(r, \alpha, t)=r, \varphi_{r}(\infty, \theta, t)=0, \varphi(r, \theta, 0)=0,
\end{gathered}
$$

where $\Delta$ stands up for the Laplace operator in polar coordinates $(r, \theta)$. We seek its solution as $\varphi=U-V$, where $U=-r \cos \theta / \sin \alpha$ is a steady solution of (A.1) and the first two conditions of (A.2). $V$ is the solution of

$$
\frac{\partial V}{\partial t}=\Delta V ; V_{\theta}(r, 0, t)=V_{\theta}(r, \alpha, t)=0, \quad V_{r}(\infty, \theta, t)=-\frac{\cos \theta}{\sin \alpha}, V(r, \theta, 0)=U .
$$

It can be written as

$$
V(r, \theta, t)=-\int_{0}^{\alpha} \int_{0}^{\infty} \rho \frac{\cos \omega}{\sin \alpha} v(r, \theta, t ; \rho, \omega) \rho d \rho d \omega
$$

in terms of the Green function $v(r, \theta, t ; \rho, \omega)$, or temperature at the instant $t$ at the point $(r, \theta)$ due to a instantaneous source of strength unity located at the point $(\rho, \omega)$. This function can be obtained [14, p. 379], using the Laplace's transform, as

$$
v(r, \theta, t ; \rho, \omega)=\frac{1}{2 \alpha t} e^{-\frac{r^{2}+\rho^{2}}{4 t}}\left[I_{0}\left(\frac{r \rho}{2 t}\right)+2 \sum_{n=1}^{\infty} \cos \frac{n \pi}{\alpha} \theta \cos \frac{n \pi}{\alpha} \omega I_{\frac{n \pi}{\alpha}}\left(\frac{r \rho}{2 t}\right)\right],
$$


where $I_{\mu}(z)$ is the modified Bessel function of order $\mu$.

Taking into account that $2 \int_{0}^{\alpha} \cos \omega \cos (n \pi \omega / \alpha) d \omega=-2(-1)^{n} \sin \alpha /\left((n \pi / \alpha)^{2}-1\right)$, the solution of (A.1)-(A.2) can be written in the form

$$
\begin{aligned}
\varphi(r, \theta, t)= & \frac{4 \sqrt{t}}{\alpha} e^{-r^{2} / 4 t} \int_{0}^{\infty} I_{0}\left(\frac{r \xi}{\sqrt{t}}\right) e^{-\xi^{2} \xi^{2} d \xi-r \frac{\cos \theta}{\sin \alpha}} \\
& -\frac{8 \sqrt{t}}{\alpha} e^{-r^{2} / 4 t} \sum_{n=1}^{\infty} \frac{(-1)^{n} \cos (n \pi \theta / \alpha)}{(n \pi / \alpha)^{2}-1} \int_{0}^{\infty} I_{\frac{n \pi}{\alpha}}\left(\frac{r \xi}{\sqrt{t}}\right) e^{-\xi^{2} \xi^{2} d \xi}
\end{aligned}
$$

For small values of $r / \sqrt{t}$ only values of $\xi$ of order unity need to be considered in the integrals appearing in (A.8). Then, taking into account the behavior of the Bessel functions for small values of their argument, the temperature distribution given by (A.6) simplifies to $\varphi=\sqrt{\pi t} / \alpha-r \cos \theta / \sin \alpha$, near the edge of the wedge. Here, the second contribution is the steady solution of the heat conduction equation accounting for the external heat flux, and the edge temperature is $\sqrt{\pi t} / \alpha$, determined by the history effects. For times close to $t=t_{I}$ we can use the relation

$$
\varphi(r, \theta, t)=\varphi_{I}+\frac{\sqrt{\pi}}{2 \alpha \sqrt{t_{I}}}\left(t-t_{I}\right)-r \frac{\cos \theta}{\sin \alpha}+\mathcal{O}\left(\left(t-t_{I}\right)^{2}, \frac{r^{2}}{t_{I}}\right)
$$

with $\varphi_{I}=\sqrt{\pi t_{I}} / \alpha$.

Notice that, although the spatial variation in (A.7) is readily found - because the heat conduction term is dominant in (A.1) for $r \ll 1-$ the edge temperature is more difficult to obtain, since it involves the evaluation of (A.6). However, this time contribution can also be easily obtained by using the method of superposition, as follows: if we designate by $\varphi_{\alpha}(r, \theta, t)$ the solution of (A.1)-(A.2), it can be seen that the solution corresponding to an angle $2 \alpha / n(n \in \mathbb{N})$ is given by

$$
\varphi_{\alpha / n}(r, \theta, t)=\sum_{k=0}^{n-1} \varphi_{\alpha}\left(r, \theta+k \frac{\alpha}{n}, t\right) .
$$

In particular $\varphi_{\alpha / n}(0, \theta, t)=n \varphi_{\alpha}(0, \theta, t)$ and $m \varphi_{m \alpha / n}(0, \theta, t)=\varphi_{\alpha / n}(0, \theta, t)(m \in \mathbb{N})$. Therefore, if we begin with the one-dimensional solution corresponding to $2 \alpha=\pi$, we ebtain $\varphi_{m \pi / 2 n}(0, \theta, t)=2 n \sqrt{\pi t} / \pi m$. Then the completeness property of $\mathbb{R}$ leads to $\varphi_{\alpha}(0, \theta, t)=\sqrt{\pi t} / \alpha$ for any real value of $\alpha$.

Appendix B. The three-dimensional square corner. With the assumptions and the notation of $\S 2.1$, here using $\varphi_{I}=6 \sqrt{t_{I} / \pi}$ and $\sigma=3\left(t-t_{I}\right) \beta / \sqrt{\pi t_{I}}\left(1+\varphi_{I}\right)^{2}$, the problem describing the ignition of a three-dimensional square corner takes the form

$$
\begin{aligned}
3 \varepsilon \frac{\partial \psi}{\partial \sigma}=\Delta \psi+\nu(\varepsilon) e^{b} \exp \left[\frac{\psi+\sigma-\xi-\eta-\zeta+\mathcal{O}(1 / \beta)}{1+\frac{1+\varphi_{I}}{\beta}(\psi+\sigma-\xi-\eta-\zeta+\mathcal{O}(1 / \beta))}\right] \\
\psi_{\xi}(0, \eta, \zeta, \sigma)=\psi_{\eta}(\xi, 0, \zeta, \sigma)=\psi_{\zeta}(\xi, \eta, 0, \sigma)=0 \\
\psi(\xi, \eta, \zeta,-\infty)=\psi_{\rho}(\rho \rightarrow \infty, \sigma)=0
\end{aligned}
$$

with $\rho=\sqrt{\xi^{2}+\eta^{2}+\zeta^{2}}$ when written in terms of the space and time variables of the reaction zone and the ignition stage.

In the ignition stage $\psi$ must be of order unity, and the time derivative term can be neglected at $\rho \sim 1$. For moderately large values of $\rho$, where the reaction term can 
be neglected, $\psi$ has the behavior

$$
\psi \sim(2 / \pi) \omega(\sigma) / \rho+C(\sigma)
$$

where $\omega(\sigma)=\nu(\varepsilon) \int_{0}^{\infty} \int_{0}^{\infty} \int_{0}^{\infty} \exp (\psi+\sigma+b-\xi-\eta-\zeta) d \xi d \eta d \zeta$. In the outer region, defined by $r=\rho \sqrt{3 \varepsilon}=\mathcal{O}(1)$, the problem (B.1)-(B.2) is reduced to the radial heat equation with a point source, located at the origin, of strength $8 \sqrt{3 \varepsilon} \omega(\sigma)$. For small values of $r$ the outer solution can be expanded as

$$
\psi \sim \frac{2}{\pi} \sqrt{3 \varepsilon} \omega(\sigma) \frac{1}{r}-\sqrt{3 \varepsilon} I(\sigma)
$$

where $I(\sigma)=\int_{-\infty}^{\sigma}(\sigma-s)^{1 / 2}(d \omega(s) / d s) d s$. The matching between (B.3) and (B.4) clearly implies $C(\sigma)=0$. Therefore, the function $\psi$ verifies the boundary condition $\psi \rightarrow 0$ as $\rho \rightarrow \infty$, if terms of order $\sqrt{\varepsilon}$ are neglected. If we want ignition to take place at $\sigma=0, \nu(\varepsilon) e^{b}$ must be of order unity; thus we can choose $\nu(\varepsilon) \equiv 1$, and then $b=\mathcal{O}(1)$. Hence, the appropriate expansions for $\psi$ and $b$ are $\psi=\psi_{0}+\sqrt{\varepsilon} \psi_{1}+\cdots$ and $b=b_{0}+\sqrt{\varepsilon} b_{1}+\cdots$, leading to the lowest-order quasi-steady problem

$$
\begin{gathered}
\Delta \psi_{0}+\delta \exp \left(\psi_{0}-\xi-\eta-\zeta\right)=0 \\
\psi_{0, \xi}(0, \eta, \zeta)=\psi_{0, \eta}(\xi, 0, \zeta)=\psi_{0, \zeta}(\xi, \eta, 0)=\psi_{0}\left(\xi^{2}+\eta^{2}+\zeta^{2} \rightarrow \infty\right)=0
\end{gathered}
$$

where $\delta=e^{\sigma+b}$. This problem, to be solved numerically, is very similar to that considered in [11]. We can expect, as in similar reactive-diffusive systems, two solutions to exist for values of $\delta$ smaller than a certain critical value, i.e., the solution branch $[\|\psi\|, \delta]$ is expected to show a turning point at some value of $\delta$, say $\delta_{I}$, at which $\partial \psi_{0} / \partial \sigma$ becomes unbounded, so that for $\delta$ greater than $\delta_{I}$ there is no solution to (B.5)-(B.6). We can identify $\delta_{I}$ as the value of $\delta$ at which the thermal runaway occurs, if terms of order $\sqrt{\varepsilon}$ are neglected. Therefore, if the ignition is chosen to take place at $t=t_{I}$, i.e., $\sigma=0$, then $b=\ln \delta_{I}$ and the following relation between $B$ and $t_{I}$ holds:

$$
B=\delta_{I}\left(\pi t_{I}\right)^{-1 / 2} \varepsilon^{-1} \exp \left(\beta /\left(1+\varphi_{I}\right)\right) .
$$

The numerical calculations shows $\delta_{I}=1.55$. Notice the order of magnitude change of the value of $B$, resulting in a given ignition temperature $\varphi_{I}$, for the threedimensional case as compared with the two-dimensional case (2.15) and the onedimensional value $B=e^{b}\left(\pi t_{I}\right)^{-1 / 2} \varepsilon^{-1 / 2} \exp \left(\beta /\left(1+\varphi_{I}\right)\right)$, with $e^{b} \simeq 0.65$, obtained in [2].

Acknowledgments. Part of Vázquez-Espí's work was carried out during a stay at the Center for Energy and Combustion Research, University of California, San Diego. Thanks are due to Prof. Williams and the AMES staff for their hospitality.

\section{REFERENCES}

[1] H. H. JR. BRADley, Theory of ignition of a reactive solid by constant energy flux, Comb. Sci. Tech., 2 (1970), pp. 11-20.

[2] A. LiÑÁN AND F. A. WILLIAMS, Theory of ignition of a reactive solid by constant energy flux, Comb. Sci. Tech., 3 (1971), pp. 91-98.

[3] ____ Ignition of a reactive solid exposed to a step in surface temperature, SIAM J. Appl. Math., 36 (1979), pp. 587-603.

[4] A. K. KAPILA, Evolution of deflagration in a cold combustible subjected to a uniform energy flux, Internat. J. Engrg. Sci., 19 (1981), pp. 495-509. 
[5] W. E. OlmStEAD, Ignition of a combustible half-space, SIAM J. Appl.Math., 43 (1983), pp. 1-15.

[6] D. G. LASSEIGNE AND W. E. OlmsteAD, Ignition of a combustible solid with reactant consumption, SIAM J. Appl. Math., 47 (1987), pp. 332-342.

[7] _ Ignition or non ignition of a combustible solid with marginal heating, Quart. Appl. Math., 49 (1991), pp. 303-312.

[8] L. G. VorsteVeld AND C. E. HeRMANCE, Effect of geometry on ignition of a reactive solid: square corner, AIAA J., 25 (1987), pp. 592-597.

[9] - Effect of geometry on ignition of a reactive solid: acute angles, J. Propulsion, 5 (1989), pp. 26-31.

[10] A. D. MARgolin, G. N. Mokhin, And V. G. Krupin, Ignition of a wedge and cone by a thermal flux in a homogeneous reaction, Combustion, Explosion and Shock Waves, 26 (1990), pp. 17-22.

[11] C. VÁZQUEZ-ESPí AND A. LIÑÁN, The effect of square corners on the ignition of solids, SIAM J. Appl. Math., 53 (1993), pp. 1567-1590.

[12] - The ignition of solids: an asymptotic analysis, preprint.

[13] J. W. Dold, Analysis of the early stage of thermal runaway, Quart. J. Mech. Appl. Math., 38 (1985), pp. 361-385.

[14] H. S. Carslaw and J. C. Jaeger, Conduction of Heat in Solids, 2nd ed., Oxford University Press, Oxford, 1965.

[15] V. S. Berman, I. S. Riazantsev And V. M. Shevtsova, Asymptotic analysis of the process of igniting a combustible gas mixture by thermal inhomogeneity, J. Appl. Math. Mech. (PMM), 43 (1981), pp. 60-64.

[16] A. LIÑÁN AND C. VÁZQUEZ-EsPí, Ignition of a gaseous reacting mixture subjected to a localized, instantaneous, energy source, preprint. 